\title{
Stochastic Optimization
}

\author{
James C. Spall
}

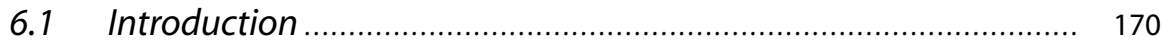

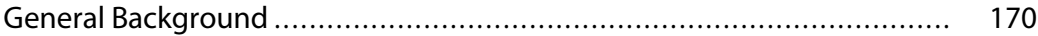

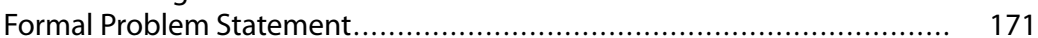

Contrast of Stochastic and Deterministic Optimization ....................... 172

Some Principles of Stochastic Optimization .................................... 174

6.2 Random Search ....................................................................... 176

Some General Properties of Direct Random Search ............................ 176

Two Algorithms for Random Search ............................................ 177

6.3 Stochastic Approximation ........................................................ 180

Introduction......................................................................... 180

Finite-Difference SA ............................................................ 181

Simultaneous Perturbation SA ................................................... 183

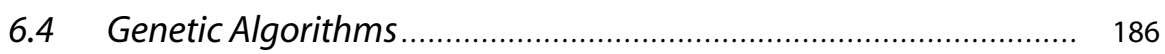

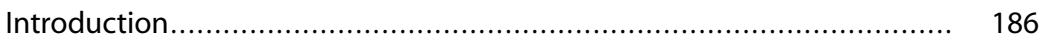

Chromosome Coding and the Basic GA Operations ........................... 188

The Core Genetic Algorithm ...................................................... 191

Some Implementation Aspects ...................................................... 191

Some Comments on the Theory for GAs ...................................... 192

6.5 Concluding Remarks ................................................................ 194 
Stochastic optimization algorithms have been growing rapidly in popularity over the last decade or two, with a number of methods now becoming "industry standard" approaches for solving challenging optimization problems. This paper provides a synopsis of some of the critical issues associated with stochastic optimization and a gives a summary of several popular algorithms. Much more complete discussions are available in the indicated references.

To help constrain the scope of this article, we restrict our attention to methods using only measurements of the criterion (loss function). Hence, we do not cover the many stochastic methods using information such as gradients of the loss function. Section 6.1 discusses some general issues in stochastic optimization. Section 6.2 discusses random search methods, which are simple and surprisingly powerful in many applications. Section 6.3 discusses stochastic approximation, which is a foundational approach in stochastic optimization. Section 6.4 discusses a popular method that is based on connections to natural evolution - genetic algorithms. Finally, Sect. 6.5 offers some concluding remarks.

\subsection{Introduction}

\subsubsection{General Background}

Stochastic optimization plays a significant role in the analysis, design, and operation of modern systems. Methods for stochastic optimization provide a means of coping with inherent system noise and coping with models or systems that are highly nonlinear, high dimensional, or otherwise inappropriate for classical deterministic methods of optimization. Stochastic optimization algorithms have broad application to problems in statistics (e.g., design of experiments and response surface modeling), science, engineering, and business. Algorithms that employ some form of stochastic optimization have become widely available. For example, many modern data mining packages include methods such as simulated annealing and genetic algorithms as tools for extracting patterns in data.

Specific applications include business (making short- and long-term investment decisions in order to increase profit), aerospace engineering (running computer simulations to refine the design of a missile or aircraft), medicine (designing laboratory experiments to extract the maximum information about the efficacy of a new drug), and traffic engineering (setting the timing for the signals in a traffic network). There are, of course, many other applications.

Let us introduce some concepts and notation. Suppose $\Theta$ is the domain of allowable values for a vector $\boldsymbol{\theta}$. The fundamental problem of interest is to find the value(s) of a vector $\boldsymbol{\theta} \in \Theta$ that minimize a scalar-valued loss function $L(\boldsymbol{\theta})$. Other common names for $L$ are performance measure, objective function, measureof-effectiveness (MOE), fitness function (or negative fitness function), or criterion. While this problem refers to minimizing a loss function, a maximization problem (e.g., maximizing profit) can be trivially converted to a minimization problem by 
changing the sign of the criterion. This paper focuses on the problem of minimization. In some cases (i.e., differentiable $L$ ), the minimization problem can be converted to a root-finding problem of finding $\boldsymbol{\theta}$ such that $\boldsymbol{g}(\boldsymbol{\theta})=\partial L(\theta) / \partial \theta=\mathbf{0}$. Of course, this conversion must be done with care because such a root may not correspond to a global minimum of $L$.

The three remaining subsections in this section define some basic quantities, discuss some contrasts between (classical) deterministic optimization and stochastic optimization, and discuss some basic properties and fundamental limits. This section provides the foundation for interpreting the algorithm presentations in Sects. 6.2 to 6.4. There are many other references that give general reviews of various aspects of stochastic optimization. Among these are Arsham (1998), Fouskakis and Draper (2002), Fu (2002), Gosavi (2003), Michalewicz and Fogel (2000), and Spall (2003).

\section{Formal Problem Statement}

The problem of minimizing a loss function $L=L(\boldsymbol{\theta})$ can be formally represented as finding the set:

$$
\Theta^{*} \equiv \arg \min _{\boldsymbol{\theta} \in \Theta} L(\boldsymbol{\theta})=\left\{\boldsymbol{\theta}^{*} \in \Theta: L\left(\boldsymbol{\theta}^{*}\right) \leq L(\boldsymbol{\theta}) \quad \text { for all } \quad \boldsymbol{\theta} \in \Theta\right\},
$$

where $\boldsymbol{\theta}$ is the $p$-dimensional vector of parameters that are being adjusted and $\Theta \subseteq \mathbb{R}^{p}$. The "arg $\min _{\theta \in \Theta}$ " statement in (6.1) should be read as: $\Theta^{*}$ is the set of values $\boldsymbol{\theta}=\boldsymbol{\theta}^{*}$ ( $\boldsymbol{\theta}$ the "argument" in "arg min") that minimize $L(\boldsymbol{\theta})$ subject to $\boldsymbol{\theta}^{*}$ satisfying the constraints represented in the set $\Theta$. The elements $\theta^{*} \in \Theta^{*} \subseteq \Theta$ are equivalent solutions in the sense that they yield identical values of the loss function. The solution set $\Theta^{*}$ in (6.1) may be a unique point, a countable (finite or infinite) collection of points, or a set containing an uncountable number of points.

For ease of exposition, this paper generally focuses on continuous optimization problems, although some of the methods may also be used in discrete problems. In the continuous case, it is often assumed that $L$ is a "smooth" (perhaps several times differentiable) function of $\boldsymbol{\theta}$. Continuous problems arise frequently in applications such as model fitting (parameter estimation), adaptive control, neural network training, signal processing, and experimental design. Discrete optimization (or combinatorial optimization) is a large subject unto itself (resource allocation, network routing, policy planning, etc.).

A major issue in optimization is distinguishing between global and local optima. All other factors being equal, one would always want a globally optimal solution to the optimization problem (i.e., at least one $\boldsymbol{\theta}^{*}$ in the set of values $\Theta^{*}$ ). In practice, however, it may not be feasible to find a global solution and one must be satisfied with obtaining a local solution. For example, $L$ may be shaped such that there is a clearly defined minimum point over a broad region of the domain $\Theta$, while there is a very narrow spike at a distant point. If the trough of this spike is lower than any point in the broad region, the local optimal solution is better than any nearby $\boldsymbol{\theta}$, but it is not be the best possible $\boldsymbol{\theta}$. 
It is usually only possible to ensure that an algorithm will approach a local minimum with a finite amount of resources being put into the optimization process. That is, it is easy to construct functions that will "fool" any known algorithm, unless the algorithm is given explicit prior information about the location of the global solution - certainly not a case of practical interest! However, since the local minimum may still yield a significantly improved solution (relative to no formal optimization process at all), the local minimum may be a fully acceptable solution for the resources available (human time, money, computer time, etc.) to be spent on the optimization. However, we discuss several algorithms (random search, stochastic approximation, and genetic algorithms) that are sometimes able to find global solutions from among multiple local solutions.

\subsubsection{Contrast of Stochastic and Deterministic Optimization}

As a paper on stochastic optimization, the algorithms considered here apply where:

I. There is random noise in the measurements of $L(\boldsymbol{\theta})$ - and/or -

II. There is a random (Monte Carlo) choice made in the search direction as the algorithm iterates toward a solution.

In contrast, classical deterministic optimization assumes that perfect information is available about the loss function (and derivatives, if relevant) and that this information is used to determine the search direction in a deterministic manner at every step of the algorithm. In many practical problems, such information is not available. We discuss properties I and II below.

Let $\hat{\boldsymbol{\theta}}_{k}$ be the generic notation for the estimate for $\boldsymbol{\theta}$ at the $k$ th iteration of whatever algorithm is being considered, $k=0,1,2, \ldots$. Throughout this paper, the specific mathematical form of $\hat{\boldsymbol{\theta}}_{k}$ will change as the algorithm being considered changes. The following notation will be used to represent noisy measurements of $L$ at a specific $\theta$ :

$$
y(\boldsymbol{\theta}) \equiv L(\boldsymbol{\theta})+\varepsilon(\boldsymbol{\theta}),
$$

where $\varepsilon$ represents the noise term. Note that the noise terms show dependence on $\boldsymbol{\theta}$ This dependence is relevant for many applications. It indicates that the common statistical assumption of independent, identically distributed (i.i.d.) noise does not necessarily apply since $\boldsymbol{\theta}$ will be changing as the search process proceeds.

Relative to property I, noise fundamentally alters the search and optimization process because the algorithm is getting potentially misleading information throughout the search process. For example, as described in Example 1.4 of Spall (2003), consider the following loss function with a scalar $\theta: L(\theta)=\mathrm{e}^{-0.1 \theta} \sin (2 \theta)$. If the domain for optimization is $\Theta=[0,7]$, the (unique) minimum occurs at $\theta^{*}=3 \pi / 4 \approx 2.36$, as shown in Fig. 6.1. Suppose that the analyst carrying out the optimization is not able to calculate $L(\theta)$, obtaining instead only noisy measurements $y(\theta)=L(\theta)+\varepsilon$, where the noises $\varepsilon$ are i.i.d. with distribution $N\left(0,0.5^{2}\right)$ 
(a normal distribution with mean zero and variance $0.5^{2}$ ). The analyst uses the $y(\theta)$ measurements in conjunction with an algorithm to attempt to find $\theta^{*}$.

Consider the experiment depicted in Fig. 6.1 (with data generated via MATLAB). Based on the simple method of collecting one measurement at each increment of 0.1 over the interval defined by $\Theta$ (including the endpoints 0 and 7), the analyst would falsely conclude that the minimum is at $\theta=5.9$. As shown, this false minimum is far from the actual $\theta^{*}$.

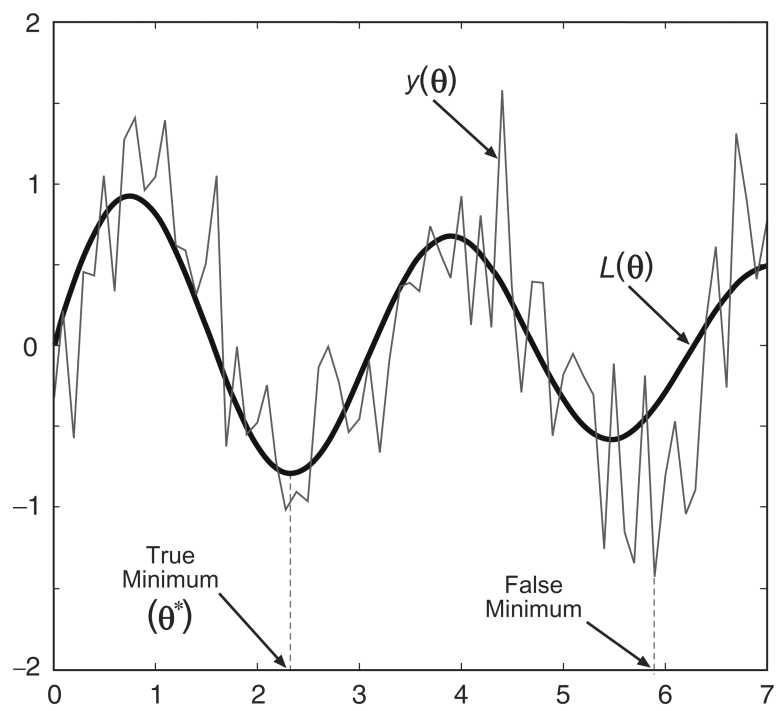

Figure 6.1. Simple loss function $L(\theta)$ with indicated minimum $\theta^{*}$. Note how noise causes the algorithm to be deceived into sensing that the minimum is at the indicated false minimum (Reprinted from Introduction to Stochastic Search and Optimization with permission of John Wiley \& Sons, Inc.)

Noise in the loss function measurements arises in almost any case where physical system measurements or computer simulations are used to approximate a steadystate criterion. Some specific areas of relevance include real-time estimation and control problems where data are collected "on the fly" as a system is operating and problems where large-scale simulations are run as estimates of actual system behavior.

Let us summarize two distinct problems involving noise in the loss function measurements: target tracking and simulation-based optimization. In the tracking problem there is a mean-squared error (MSE) criterion of the form

$$
L(\boldsymbol{\theta})=E\left(\| \text { actual output }- \text { desired output } \|^{2}\right) .
$$

The stochastic optimization algorithm uses the actual (observed) squared error $y(\boldsymbol{\theta})=\|\cdot\|^{2}$, which is equivalent to an observation of $L$ embedded in noise. In the simulation problem, let $L(\boldsymbol{\theta})$ be the loss function representing some type of 
"average" performance for the system. A single run of a Monte Carlo simulation at a specific value of $\boldsymbol{\theta}$ provides a noisy measurement: $y(\boldsymbol{\theta})=L(\boldsymbol{\theta})+$ noise at $\boldsymbol{\theta}$. (Note that it is rarely desirable to spend computational resources in averaging many simulation runs at a given value of $\boldsymbol{\theta}$; in optimization, it is typically necessary to consider many values of $\boldsymbol{\theta}$.) The above problems are described in more detail in Examples 1.5 and 1.6 in Spall (2003).

Relative to the other defining property of stochastic optimization, property II (i.e., randomness in the search direction), it is sometimes beneficial to deliberately introduce randomness into the search process as a means of speeding convergence and making the algorithm less sensitive to modeling errors. This injected (Monte Carlo) randomness is usually created via computer-based pseudorandom number generators. One of the roles of injected randomness in stochastic optimization is to allow for "surprise" movements to unexplored areas of the search space that may contain an unexpectedly good $\boldsymbol{\theta}$ value. This is especially relevant in seeking out a global optimum among multiple local solutions. Some algorithms that use injected randomness are random search (Sect. 6.2), simultaneous perturbation stochastic approximation (Sect. 6.3), and genetic algorithms (Sect. 6.4).

\subsubsection{Some Principles of Stochastic Optimization}

The discussion above is intended to motivate some of the issues and challenges in stochastic optimization. Let us now summarize some important issues for the implementation and interpretation of results in stochastic optimization.

The first issue we mention is the fundamental limits in optimization with only noisy information about the $L$ function. Foremost, perhaps, is that the statistical error of the information fed into the algorithm - and the resulting error of the output of the algorithm - can only be reduced by incurring a significant cost in number of function evaluations. For the simple case of independent noise, the error decreases at the rate $1 / \sqrt{N}$, where $N$ represents the number of $L$ measurements fed into the algorithm. This is a classical result in statistics, indicating that a 25 -fold increase in function evaluations reduces the error by a factor of five.

A further limit for multivariate $(p>1)$ optimization is that the volume of the search region generally grows rapidly with dimension. This implies that one must usually exploit problem structure to have a hope of getting a reasonable solution in a high-dimensional problem.

All practical problems involve at least some restrictions on $\boldsymbol{\theta}$, although in some applications it may be possible to effectively ignore the constraints. Constraints can be encountered in many different ways, as motivated by the specific application. Note that the constraint set $\Theta$ does not necessarily correspond to the set of allowable values for $\boldsymbol{\theta}$ in the search since some problems allow for the "trial" values of the search to be outside the set of allowable final estimates. Constraints are usually handled in practice on an ad hoc basis, especially tuned to the problem at hand. There are few general, practical methods that apply broadly in stochastic optimization. Michalewicz and Fogel (2000, Chap. 9), for 
example, discuss some of the practical methods by which constraints are handled in evolutionary computation. Similar methods apply in other stochastic algorithms.

In general search and optimization, it is very difficult (perhaps impossible) to develop automated methods for indicating when the algorithm is close enough to the solution that it can be stopped. Without prior knowledge, there is always the possibility that $\boldsymbol{\theta}^{*}$ could lie in some unexplored region of the search space. This applies even when the functions involved are relatively benign; see Solis and Wets (1981) for mention of this in the context of twice-differentiable convex $L$. Difficulties are compounded when the function measurements include noise.

It is quite normal for the environment to change over time. Hence, the solution to a problem now may not be the best (or even a good) solution to the corresponding problem in the future. In some search and optimization problems, the algorithm will be explicitly designed to adapt to a changing environment and automatically provide a new estimate at the optimal value (e.g., a control system). In other cases, one needs to restart the process and find a new solution. In either sense, the problem solving may never stop!

In reading or contributing to the literature on stochastic optimization, it is important to recognize the limits of numerical comparisons by Monte Carlo. Monte Carlo studies can be a sound scientific method of gaining insight and can be a useful supplement to theory, much of which is based on asymptotic (infinite sample) analysis. In fact, it is especially popular in certain branches of optimization to create "test suites" of problems, where various algorithms compete against each other. A danger arises, however, in making broad claims about the performance of individual algorithms based on the results of numerical studies. Performance can vary tremendously under even small changes in the form of the functions involved or the coefficient settings within the algorithms themselves. One must be careful about drawing conclusions beyond those directly supported by the specific numerical studies performed. For purposes of drawing objective conclusions about the relative performance of algorithms, it is preferable to use both theory and numerical studies.

Some real systems have one (unique) globally "best" operating point $\left(\boldsymbol{\theta}^{*}\right)$ in the domain $\Theta$ while others have multiple global solutions (in either case, of course, there could be many locally optimal solutions). To avoid excessively cumbersome discussion of algorithms and supporting implementation issues and theory, we will often refer to "the" solution $\boldsymbol{\theta}^{*}$ (versus "a" solution $\boldsymbol{\theta}^{*}$ ). In practice, an analyst may be quite satisfied to reach a solution at or close to any one $\theta^{*} \in \Theta^{*}$.

The so-called no free lunch (NFL) theorems provide a formal basis for the intuitively appealing idea that there is a fundamental tradeoff between algorithm efficiency and algorithm robustness (reliability and stability in a broad range of problems). In essence, algorithms that are very efficient on one type of problem are not automatically efficient on problems of a different type. Hence, there can never be a universally best search algorithm just as there is rarely (never?) a universally best solution to any general problem of society. Wolpert and Macready (1997) 
provided a general formal structure for the NFL theorems, although the general ideas had been around for a long time prior to their paper (Wolpert and Macready were the ones to coin the expression "no free lunch" in this search and optimization context). The NFL theorems are established for discrete optimization with a finite (but arbitrarily large) number of options. However, their applicability includes most practical continuous problems because virtually all optimization is carried out on 32- or 64-bit digital computers. The theorems apply to the cases of both noise-free and noisy loss measurements. NFL states, in essence, that an algorithm that is effective on one class of problems is guaranteed to be ineffective on another class. Spall (2003, Sects. 1.2.2 and 10.6) provides more-detailed discussion on the basis and implications of NFL.

We are now in a position to discuss several popular stochastic optimization methods. The summaries here are just that - summaries. Much more complete discussions are available in the indicated references or in Spall (2003). We let $\hat{\boldsymbol{\theta}}_{k}$ represent the estimate for $\boldsymbol{\theta}$ at the $k$ th iteration of an algorithm under consideration. Section 6.2 discusses random search methods, which are simple and surprisingly powerful in many applications. Section 6.3 discusses stochastic approximation and Sect. 6.4 discusses the popular genetic algorithms. Because of the relative brevity of this review, there are many methods of stochastic optimization not covered here, including simulated annealing, stochastic programming, evolutionary computation other than genetic algorithms, temporal difference methods, and so on. Readers with an interest in one of those may consult the references listed at the end of Sect. 6.1.1.

\subsection{Random Search}

This section describes some simple methods based on the notion of randomly searching over the domain of interest. Section 6.2.1 gives a short discussion of general issues in direct random search methods. The algorithms discussed in Sect. 6.2.2 represent two versions of random search.

\subsubsection{Some General Properties of Direct Random Search}

Consider the problem of trying to find the optimal $\theta \in \Theta$ based on noise-free measurements of $L=L(\boldsymbol{\theta})$. Random search methods are perhaps the simplest methods of stochastic optimization in such a setting and can be quite effective in many problems. Their relative simplicity is an appealing feature to both practitioners and theoreticians. These direct random search methods have a number of advantages relative to most other search methods. The advantages include relative ease of coding in software, the need to only obtain $L$ measurements (versus gradients or other ancillary information), reasonable computational efficiency (especially for those direct search algorithms that make use of some local information in their search), broad applicability to non-trivial loss functions and/or to $\theta$ that may be 
continuous, discrete, or some hybrid form, and a strong theoretical foundation. Some of these attributes were mentioned in the forward-looking paper of Karnopp (1963). A good recent survey of random search and related methods is Kolda et al. (2003).

\section{Two Algorithms for Random Search}

This section describes two direct random search techniques. These two algorithms represent only a tiny fraction of available methods. Solis and Wets (1981) and Zhigljavsky (1991) are among many references discussing these and other random search methods. The two algorithms here are intended to convey the essential flavor of most available direct random search algorithms. With the exception of some discussion at the end of the subsection, the methods here assume perfect (noise-free) values of $L$.

The first method we discuss is "blind random search." This is the simplest random search method, where the current sampling for $\boldsymbol{\theta}$ does not take into account the previous samples. That is, this blind search approach does not adapt the current sampling strategy to information that has been garnered in the search process. The approach can be implemented in batch (non-recursive) form simply by laying down a number of points in $\Theta$ and taking the value of $\boldsymbol{\theta}$ yielding the lowest $L$ value as our estimate of the optimum. The approach can be conveniently implemented in recursive form as we illustrate below.

The simplest setting for conducting the random sampling of new (candidate) values of $\boldsymbol{\theta}$ is when $\Theta$ is a hypercube and we are using uniformly generated values of $\boldsymbol{\theta}$. The uniform distribution is continuous or discrete for the elements of $\boldsymbol{\theta}$ depending on the definitions for these elements. In fact, the blind search form of the algorithm is unique among all general stochastic optimization algorithms in that it is the only one without any adjustable algorithm coefficients that need to be "tuned" to the problem at hand. (Of course, a de facto tuning decision has been made by choosing the uniform distribution for sampling.)

For a domain $\Theta$ that is not a hypercube or for other sampling distributions, one may use transformations, rejection methods, or Markov chain Monte Carlo to generate the sample $\boldsymbol{\theta}$ values (see, e.g., Gentle, 2003). For example, if $\Theta$ is an irregular shape, one can generate a sample on a hypercube superset containing $\Theta$ and then reject the sample point if it lies outside of $\Theta$.

The steps for a recursive implementation of blind random search are given below. This method applies when $\boldsymbol{\theta}$ has continuous, discrete, or hybrid elements.

\section{Blind Random Search}

Step o (Initialization) Choose an initial value of $\boldsymbol{\theta}$, say $\hat{\boldsymbol{\theta}}_{0} \in \Theta$, either randomly or deterministically. (If random, usually a uniform distribution on $\Theta$ is used.) Calculate $L\left(\hat{\boldsymbol{\theta}}_{0}\right)$. Set $k=0$.

Step 1 Generate a new independent value $\boldsymbol{\theta}_{\text {new }}(k+1) \in \Theta$, according to the chosen probability distribution. If $L\left(\boldsymbol{\theta}_{\text {new }}(k+1)\right)<L\left(\hat{\boldsymbol{\theta}}_{k}\right)$, set $\hat{\boldsymbol{\theta}}_{k+1}=\boldsymbol{\theta}_{\text {new }}(k+1)$. Else, take $\hat{\boldsymbol{\theta}}_{k+1}=\hat{\boldsymbol{\theta}}_{k}$. 
Step 2 Stop if the maximum number of $L$ evaluations has been reached or the user is otherwise satisfied with the current estimate for $\boldsymbol{\theta}$ via appropriate stopping criteria; else, return to Step 1 with the new $k$ set to the former $k+1$.

The above algorithm converges almost surely (a.s.) to $\boldsymbol{\theta}^{*}$ under very general conditions (see, e.g., Spall, 2003, pp. 40-41). Of course, convergence alone is an incomplete indication of the performance of the algorithm. It is also of interest to examine the rate of convergence. The rate is intended to tell the analyst how close $\hat{\boldsymbol{\theta}}_{k}$ is likely to be to $\boldsymbol{\theta}^{*}$ for a given cost of search. While blind random search is a reasonable algorithm when $\boldsymbol{\theta}$ is low dimensional, it can be shown that the method is generally a very slow algorithm for even moderately dimensioned $\boldsymbol{\theta}$ (see, e.g., Spall, 2003, 42-43). This is a direct consequence of the exponential increase in the size of the search space as $p$ increases. As an illustration, Spall (2003, Example 2.2) considers a case where $\Theta=[0,1]^{p}$ (the $p$-dimensional hypercube with minimum and maximum values of 0 and 1 for each component of $\theta$ ) and where one wishes to guarantee with probability 0.90 that each element of $\boldsymbol{\theta}$ is within 0.04 units of the optimal value. As $p$ increases from one to ten, there is an approximate $10^{10}$-fold increase in the number of loss function evaluations required.

Blind search is the simplest random search in that the sampling generating the new $\boldsymbol{\theta}$ value does not take account of where the previous estimates of $\boldsymbol{\theta}$ have been. The random search algorithm below is slightly more sophisticated in that the random sampling is a function of the position of the current best estimate for $\boldsymbol{\theta}$. In this way, the search is more localized in the neighborhood of that estimate, allowing for a better exploitation of information that has previously been obtained about the shape of the loss function.

The localized algorithm is presented below. This algorithm was described in Matyas (1965). Note that the use of the term "localized" here pertains to the sampling strategy and does not imply that the algorithm is only useful for local (versus global) optimization in the sense described in Sect. 6.1. In fact, the algorithm has global convergence properties as described below. As with blind search, the algorithm may be used for continuous or discrete problems.

\section{Localized Random Search}

Step o (Initialization) Pick an initial guess $\hat{\boldsymbol{\theta}}_{0} \in \Theta$, either randomly or with prior information. Set $k=0$.

Step 1 Generate an independent random vector $\boldsymbol{d}_{k} \in \mathbb{R}^{p}$ and add it to the current $\boldsymbol{\theta}$ value, $\hat{\boldsymbol{\theta}}_{k}$. Check if $\hat{\boldsymbol{\theta}}_{k}+\boldsymbol{d}_{k} \in \Theta$. If $\hat{\boldsymbol{\theta}}_{k}+\boldsymbol{d}_{k} \notin \Theta$, generate a new $\boldsymbol{d}_{k}$ and repeat or, alternatively, move $\hat{\boldsymbol{\theta}}_{k}+\boldsymbol{d}_{k}$ to the nearest valid point within $\Theta$. Let $\boldsymbol{\theta}_{\text {new }}(k+1)$ equal $\hat{\boldsymbol{\theta}}_{k}+\boldsymbol{d}_{k} \in \Theta$ or the aforementioned nearest valid point in $\Theta$. Step 2 If $L\left(\theta_{\text {new }}(k+1)\right)<L\left(\hat{\boldsymbol{\theta}}_{k}\right)$, set $\hat{\boldsymbol{\theta}}_{k+1}=\boldsymbol{\theta}_{\text {new }}(k+1)$; else, set $\hat{\boldsymbol{\theta}}_{k+1}=\hat{\boldsymbol{\theta}}_{k}$.

Step 3 Stop if the maximum number of $L$ evaluations has been reached or the user is otherwise satisfied with the current estimate for $\boldsymbol{\theta}$ via appropriate stopping criteria; else, return to Step 1 with the new $k$ set to the former $k+1$. 
For continuous problems, Matyas (1965) and others have used the (multivariate) normal distribution for generating $\boldsymbol{d}_{k}$. However, the user is free to set the distribution of the deviation vector $\boldsymbol{d}_{k}$. The distribution should have mean zero and each component should have a variation (e.g., standard deviation) consistent with the magnitudes of the corresponding $\theta$ elements. This allows the algorithm to assign roughly equal weight to each of the components of $\boldsymbol{\theta}$ as it moves through the search space. Although not formally allowed in the convergence theory, it is often advantageous in practice if the variability in $\boldsymbol{d}_{k}$ is reduced as $k$ increases. This allows one to focus the search more tightly as evidence is accrued on the location of the solution (as expressed by the location of our current estimate $\hat{\theta}_{k}$ ).

The convergence theory for the localized algorithms tends to be more restrictive than the theory for blind search. Solis and Wets (1981) provide a theorem for global convergence of localized algorithms, but the theorem conditions may not be verifiable in practice. An earlier theorem from Matyas (1965) (with proof corrected in Baba et al., 1977) provides for global convergence of the localized search above if $L$ is a continuous function. The convergence is in the "in probability" sense. The theorem allows for more than one global minimum to exist in $\Theta$. Therefore, in general, the result provides no guarantee of $\hat{\boldsymbol{\theta}}_{k}$ ever settling near any one value $\boldsymbol{\theta}^{*}$. We present the theorem statement below.

Convergence Theorem for Localized Search. Let $\Theta^{*}$ represent the set of global minima for $L$ (see Sect. 6.1). Suppose that $L$ is continuous on a bounded domain $\Theta$ and that if $\hat{\boldsymbol{\theta}}_{k}+\boldsymbol{d}_{k} \notin \Theta$ at a given iteration, a new $\boldsymbol{d}_{k}$ is randomly generated. For any $\eta>0$, let $R_{\eta}=\bigcup_{\theta^{*} \in \Theta^{*}}\left\{\boldsymbol{\theta}:\left|L(\boldsymbol{\theta})-L\left(\boldsymbol{\theta}^{*}\right)\right|<\eta\right\}$. Then, for $\boldsymbol{d}_{k}$ having an i.i.d. $N\left(\mathbf{0}, \boldsymbol{I}_{p}\right)$ distribution, $\lim _{k \rightarrow \infty} P\left(\hat{\boldsymbol{\theta}}_{k} \in R_{\eta}\right)=1$.

The above algorithm might be considered the most naïve of the localized random search algorithms. More sophisticated approaches are also easy to implement. For instance, if a search in one direction increases $L$, then it is likely to be beneficial to move in the opposite direction. Further, successive iterations in a direction that tend to consistently reduce $L$ should encourage further iterations in the same direction. Many algorithms exploiting these simple properties exist (e.g., Solis and Wets, 1981, and Zhigljavsky, 1991).

In spite of its simplicity, the localized search algorithm is surprisingly effective in a wide range of problems. Several demonstrations are given in Sects. 2.2.3 and 2.3 in Spall (2003).

The random search algorithms above are usually based on perfect (noise-free) measurements of the loss function. This is generally considered a critical part of such algorithms (Pflug, 1996, p. 25). In contrast to the noise-free case, random search methods with noisy loss evaluations of the form $y(\boldsymbol{\theta})=L(\boldsymbol{\theta})+\varepsilon(\boldsymbol{\theta})$ generally do not formally converge. However, there are means by which the random search techniques can be modified to accommodate noisy measurements, at least on a heuristic basis.

Some of the limited formal convergence theory for random search as applied to the noisy measurement case includes Yakowitz and Fisher (1973, Sect. 4) and 
Zhigljavsky (1991, Chap. 3). Spall (2003, Sect. 2.3) discusses some practical methods for coping with noise, including simple averaging of the noisy loss function evaluations $y(\boldsymbol{\theta})$ at each value of $\boldsymbol{\theta}$ generated in the search process and a modification of the algorithm's key decision criterion (step 1 of blind random search and step 2 of localized random search) to build in some robustness to the noise. However, the averaging method can be costly since the error decreases only at the rate of $1 / \sqrt{N}$ when averaging $N$ function evaluations with independent noise. Likewise, the altered threshold may be costly by rejecting too many changes in $\theta$ due to the conservative nature of the modified criterion. The presence of noise in the loss evaluations makes the optimization problem so much more challenging that there is little choice but to accept these penalties if one wants to use a simple random search. We will see in the next section that stochastic approximation tends to be more adept at coping with noise at the price of a more restrictive problem setting than the noise-free convergence theorem above.

\subsection{Stochastic Approximation}

\subsubsection{Introduction}

Stochastic approximation (SA) is a cornerstone of stochastic optimization. Robbins and Monro (1951) introduced SA as a general root-finding method when only noisy measurements of the underlying function are available. Let us now discuss some aspects of SA as applied to the more specific problem of root-finding in the context of optimization. With a differentiable loss function $L(\boldsymbol{\theta})$, recall the familiar set of $p$ equations and $p$ unknowns for use in finding a minimum $\boldsymbol{\theta}^{*}$ :

$$
\boldsymbol{g}(\boldsymbol{\theta})=\frac{\partial L}{\partial \boldsymbol{\theta}}=\mathbf{0} .
$$

(Of course, side conditions are required to guarantee that a root of (6.3) is a minimum, not a maximum or saddlepoint.) Note that (6.3) is nominally only directed at local optimization problems, although some extensions to global optimization are possible, as briefly discussed in Sect. 6.3.3. There are a number of approaches for solving the problem represented by (6.3) when direct (usually noisy) measurements of the gradient $g$ are available. These typically go by the name of stochastic gradient methods (e.g., Spall, 2003, Chap. 5). In contrast to the stochastic gradient approach - but consistent with the emphasis in the random search and genetic algorithms (Sects. 6.2 and 6.4 here) - let us focus on SA when only measurements of $L$ are available. However, unlike the emphasis in random search and genetic algorithms, we consider noisy measurements of $L$.

To motivate the general SA approach, first recall the familiar form for the unconstrained deterministic steepest descent algorithm for solving (6.3):

$$
\hat{\boldsymbol{\theta}}_{k+1}=\hat{\boldsymbol{\theta}}_{k}-a_{k} \boldsymbol{g}\left(\hat{\boldsymbol{\theta}}_{k}\right),
$$


where the gain (or step size) satisfies $a_{k}>0$ (see, e.g., Bazaraa et al., 1993, pp. 300-308 or any other book on mathematical programming; Spall, 2003, Sect. 1.4). This algorithm requires exact knowledge of $g$. Steepest descent will converge to $\boldsymbol{\theta}^{*}$ under certain fairly general conditions. (A notable variation of steepest descent is the Newton-Raphson algorithm [sometimes called Newton's method; e.g., Bazaraa et al., 1993, pp. 308-312], which has the form $\hat{\boldsymbol{\theta}}_{k+1}=\hat{\boldsymbol{\theta}}_{k}-$ $a_{k} \boldsymbol{H}\left(\hat{\boldsymbol{\theta}}_{k}\right)^{-1} \boldsymbol{g}\left(\hat{\boldsymbol{\theta}}_{k}\right)$, where $\boldsymbol{H}(\cdot)$ is the Hessian (second derivative) matrix of $L$. Under more restrictive conditions, the Newton-Raphson algorithm has a much faster rate of convergence to $\boldsymbol{\theta}^{*}$ than steepest descent. However, with its requirement for a Hessian matrix, it is generally more challenging to implement. An SA version of Newton-Raphson is discussed briefly at the end of Sect. 6.3.3.)

Unlike with steepest descent, it is assumed here that we have no direct knowledge of $g$. The recursive procedure of interest is in the general SA form

$$
\hat{\boldsymbol{\theta}}_{k+1}=\hat{\boldsymbol{\theta}}_{k}-a_{k} \hat{\boldsymbol{g}}_{k}\left(\hat{\boldsymbol{\theta}}_{k}\right),
$$

where $\hat{\boldsymbol{g}}_{k}\left(\hat{\boldsymbol{\theta}}_{k}\right)$ is the estimate of $\boldsymbol{g}$ at the iterate $\hat{\boldsymbol{\theta}}_{k}$ based on measurements of the loss function. Hence, (6.4) is analogous to the steepest descent algorithm, with the gradient estimate $\hat{\boldsymbol{g}}_{k}(\boldsymbol{\theta})$ replacing the direct gradient $\boldsymbol{g}$ at $\boldsymbol{\theta}=\hat{\boldsymbol{\theta}}_{k}$. The gain $a_{k}>0$ here also acts in a way similar to its role in the steepest descent form. Under appropriate conditions, the iteration in (6.4) converges to $\boldsymbol{\theta}^{*}$ in some stochastic sense (usually almost surely, a.s.). (There are constrained forms of SA, but we do not discuss those here; see, e.g., Spall, 2003, Chaps. 4-7).

Sections 6.3.2 and 6.3.3 discuss two SA methods for carrying out the optimization task using noisy measurements of the loss function. Section 6.3.2 discusses the traditional finite-difference SA method and Sect. 6.3.3 discusses the more recent simultaneous perturbation method.

\section{Finite-Difference SA}

The essential part of (6.4) is the gradient approximation $\hat{\boldsymbol{g}}_{k}\left(\hat{\boldsymbol{\theta}}_{k}\right)$. The traditional means of forming the approximation is the finite-difference method. Expression (6.4) with this approximation represents the finite-difference SA (FDSA) algorithm. One-sided gradient approximations involve measurements $y\left(\hat{\boldsymbol{\theta}}_{k}\right)$ and $y\left(\hat{\boldsymbol{\theta}}_{k}+\right.$ perturbation $)$, while two-sided approximations involve measurements of the form $y\left(\hat{\boldsymbol{\theta}}_{k} \pm\right.$ perturbation $)$. The two-sided FD approximation for use with (6.4) is

$$
\hat{\boldsymbol{g}}_{k}\left(\hat{\boldsymbol{\theta}}_{k}\right)=\left[\begin{array}{c}
\frac{y\left(\hat{\boldsymbol{\theta}}_{k}+c_{k} \xi_{1}\right)-y\left(\hat{\boldsymbol{\theta}}_{k}-c_{k} \boldsymbol{\xi}_{1}\right)}{2 c_{k}} \\
\vdots \\
\frac{y\left(\hat{\boldsymbol{\theta}}_{k}+c_{k} \xi_{p}\right)-y\left(\hat{\boldsymbol{\theta}}_{k}-c_{k} \boldsymbol{\xi}_{p}\right)}{2 c_{k}}
\end{array}\right],
$$

where $\boldsymbol{\xi}_{i}$ denotes a vector with a 1 in the ith place and 0's elsewhere and $c_{k}>0$ defines the difference magnitude. The pair $\left\{a_{k}, c_{k}\right\}$ are the gains (or gain sequences) 
for the FDSA algorithm. The two-sided form in (6.5) is the obvious multivariate extension of the scalar two-sided form in Kiefer and Wolfowitz (1952). The initial multivariate method in Blum (1954) used a one-sided approximation.

It is of fundamental importance to determine conditions such that $\hat{\boldsymbol{\theta}}_{k}$ as shown in (6.4) and (6.5) converges to $\theta^{*}$ in some appropriate stochastic sense. The convergence theory for the FDSA algorithm is similar to "standard" convergence theory for the root-finding SA algorithm of Robbins and Monro (1951). Additional difficulties, however, arise due to a bias in $\hat{\boldsymbol{g}}_{k}\left(\hat{\boldsymbol{\theta}}_{k}\right)$ as an estimator of $\boldsymbol{g}\left(\hat{\boldsymbol{\theta}}_{k}\right)$. That is, standard conditions for convergence of SA require unbiased estimates of $\boldsymbol{g}(\cdot)$ at all $k$. On the other hand, $\hat{\boldsymbol{g}}_{k}\left(\hat{\boldsymbol{\theta}}_{k}\right)$, as shown in (6.5), is a biased estimator, with the bias having a magnitude of order $c_{k}^{2}$. We will not present the details of the convergence theory here, as it is available in many other references (e.g., Fabian, 1971; Kushner and Yin, 1997, Sects. 5.3, 8.3, and 10.3; Ruppert, 1991; Spall, 2003, Chap. 6). However, let us note that the standard conditions on the gain sequences are: $a_{k}>0, c_{k}>0, a_{k} \rightarrow 0$, $c_{k} \rightarrow 0, \sum_{k=0}^{\infty} a_{k}=\infty$, and $\sum_{k=0}^{\infty} a_{k}^{2} / c_{k}^{2}<\infty$. The choice of these gain sequences is critical to the performance of the method. Common forms for the sequences are:

$$
a_{k}=\frac{a}{(k+1+A)^{\alpha}} \quad \text { and } \quad c_{k}=\frac{c}{(k+1)^{\gamma}},
$$

where the coefficients $a, c, \alpha$, and $\gamma$ are strictly positive and $A \geq 0$. The user must choose these coefficients, a process usually based on a combination of the theoretical restrictions above, trial-and-error numerical experimentation, and basic problem knowledge. In some cases, it is possible to partially automate the selection of the gains (see, e.g., Spall, 2003, Sect. 6.6).

Let us summarize a numerical example based on the following $p=10$ loss function:

$$
L(\boldsymbol{\theta})=\boldsymbol{\theta}^{T} \boldsymbol{B}^{T} \boldsymbol{B} \boldsymbol{\theta}+0.1 \sum_{i=1}^{10}(\boldsymbol{B} \boldsymbol{\theta})_{i}^{3}+0.01 \sum_{i=1}^{10}(\boldsymbol{B} \boldsymbol{\theta})_{i}^{4},
$$

where $(\cdot)_{i}$ represents the $i$ th component of the argument vector $\boldsymbol{B} \boldsymbol{\theta}$, and $\boldsymbol{B}$ is such that $10 \boldsymbol{B}$ is an upper triangular matrix of 1's. The minimum occurs at $\boldsymbol{\theta}^{*}=\mathbf{0}$ with $L\left(\boldsymbol{\theta}^{*}\right)=0$; all runs are initialized at $\hat{\boldsymbol{\theta}}_{0}=[1,1, \ldots, 1]^{T}$ (so $L\left(\hat{\boldsymbol{\theta}}_{0}\right)=4.178$ ). Suppose that the measurement noise $\varepsilon$ is independent, identically distributed (i.i.d.) $N(0,1)$. All iterates $\hat{\boldsymbol{\theta}}_{k}$ are constrained to be in $\Theta=[-5,5]^{10}$. If an iterate falls outside of $\Theta$, each individual component of the candidate $\boldsymbol{\theta}$ that violates the interval $[-5,5]$ is mapped to it nearest endpoint \pm 5 . The subsequent gradient estimate is formed at the modified (valid) $\boldsymbol{\theta}$ value. (The perturbed values $\hat{\boldsymbol{\theta}}_{k} \pm c_{k} \boldsymbol{\xi}_{i}$ are allowed to go outside of $\Theta$.)

Using $n=1000$ loss measurements per run, we compare FDSA with the localized random search method of Sect. 6.2. Based on principles for gain selection in Spall (2003, Sect. 6.6) together with some limited trial-and-error experimentation, we chose $a=0.5, c=1, A=5, \alpha=0.602$, and $\gamma=0.101$ for FDSA and an average of 20 loss measurements per iteration with normally distributed perturbations having distribution $N\left(0,0.5^{2} \boldsymbol{I}_{10}\right)$ for the random search method. 
Figure 6.2 summarizes the results. Each curve represents the sample mean of 50 independent replications. An individual replication of one of the two algorithms has much more variation than the corresponding smoothed curve in the figure.

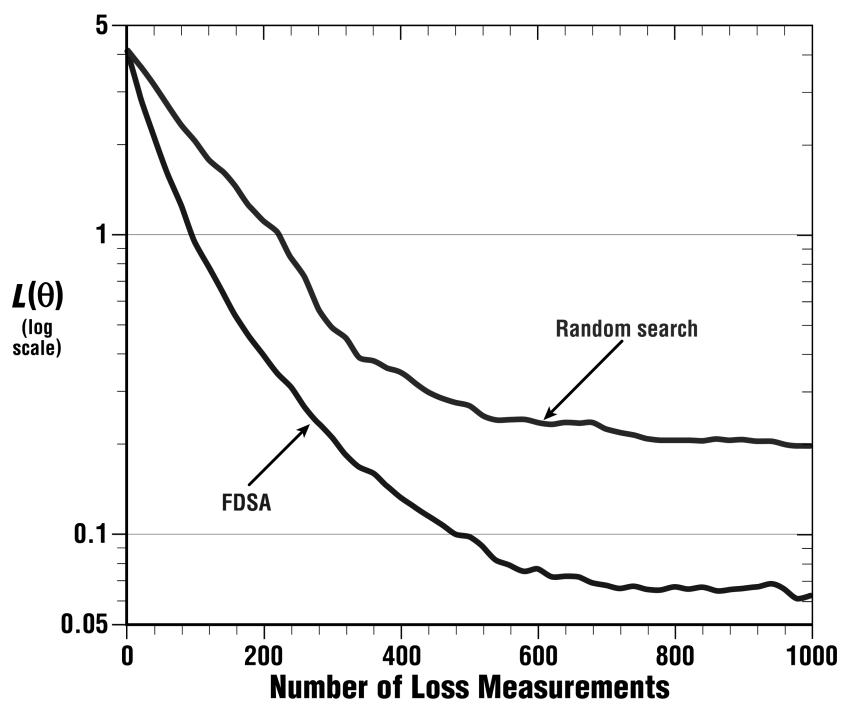

Figure 6.2. Comparison of FDSA and localized random search. Each curve represents sample mean of 50 independent replications

Figure 6.2 shows that both algorithms produce an overall reduction in the (true) loss function as the number of measurements approach 1000. The curves illustrate that FDSA outperforms random search in this case. To make the comparison fair, attempts were made to tune each algorithm to provide approximately the best performance possible. Of course, one must be careful about using this example to infer that such a result will hold in other problems as well.

\section{Simultaneous Perturbation SA}

The FDSA algorithm of Sect. 6.3.2 is a standard SA method for carrying out optimization with noisy measurement of the loss function. However, as the dimension $p$ grows large, the number of loss measurements required may become prohibitive. That is, each two-sided gradient approximation requires $2 p$ loss measurements. More recently, the simultaneous perturbation SA (SPSA) method was introduced, requiring only two measurements per iteration to form a gradient approximation independent of the dimension $p$. This provides the potential for a large savings in the overall cost of optimization.

Beginning with the generic SA form in (6.4), we now present the SP form of the gradient approximation. In this form, all elements of $\hat{\boldsymbol{\theta}}_{k}$ are randomly perturbed 
together to obtain two loss measurements $y(\cdot)$. For the two-sided SP gradient approximation, this leads to

$$
\begin{aligned}
\hat{\boldsymbol{g}}_{k}\left(\hat{\boldsymbol{\theta}}_{k}\right) & =\left[\begin{array}{c}
\frac{y\left(\hat{\boldsymbol{\theta}}_{k}+c_{k} \boldsymbol{\Delta}_{k}\right)-y\left(\hat{\boldsymbol{\theta}}_{k}-c_{k} \Delta_{k}\right)}{2 c_{k} \Delta_{k 1}} \\
\vdots \\
\frac{y\left(\hat{\boldsymbol{\theta}}_{k}+c_{k} \boldsymbol{\Delta}_{k}\right)-y\left(\hat{\boldsymbol{\theta}}_{k}-c_{k} \boldsymbol{\Delta}_{k}\right)}{2 c_{k} \Delta_{k p}}
\end{array}\right] \\
& =\frac{y\left(\hat{\boldsymbol{\theta}}_{k}+c_{k} \boldsymbol{\Delta}_{k}\right)-y\left(\hat{\boldsymbol{\theta}}_{k}-c_{k} \boldsymbol{\Delta}_{k}\right)}{2 c_{k}}\left[\Delta_{k 1}^{-1}, \Delta_{k 2}^{-1}, \ldots, \Delta_{k p}^{-1}\right]^{T},
\end{aligned}
$$

where the mean-zero $p$-dimensional random perturbation vector, $\Delta_{k}=\left[\Delta_{k 1}, \Delta_{k 2}\right.$, $\left.\ldots, \Delta_{k p}\right]^{T}$, has a user-specified distribution satisfying certain conditions and $c_{k}$ is a positive scalar (as with FDSA). Because the numerator is the same in all $p$ components of $\hat{\boldsymbol{g}}_{k}\left(\hat{\boldsymbol{\theta}}_{k}\right)$, the number of loss measurements needed to estimate the gradient in SPSA is $t w o$, regardless of the dimension $p$.

Relative to FDSA, the $p$-fold measurement savings per iteration, of course, provides only the potential for SPSA to achieve large savings in the total number of measurements required to estimate $\boldsymbol{\theta}$ when $p$ is large. This potential is realized if the number of iterations required for effective convergence to an optimum $\boldsymbol{\theta}^{*}$ does not increase in a way to cancel the measurement savings per gradient approximation. One can use asymptotic distribution theory to address this issue. In particular, both FDSA and SPSA are known to be asymptotically normally distributed under very similar conditions. One can use this asymptotic distribution result to characterize the mean-squared error $E\left(\left\|\hat{\boldsymbol{\theta}}_{k}-\boldsymbol{\theta}^{*}\right\|^{2}\right)$ for the two algorithms for large $k$. Fortunately, under fairly broad conditions, the $p$-fold savings at each iteration is preserved across iterations. In particular, based on asymptotic considerations:

Under reasonably general conditions (see Spall, 1992, or Spall, 2003, Chap. 7), the SPSA and FDSA algorithms achieve the same level of statistical accuracy for a given number of iterations even though SPSA uses only $1 / p$ times the number of function evaluations of FDSA (since each gradient approximation uses only $1 / p$ the number of function evaluations).

The SPSA Web site (www.jhuapl.edu/SPSA) includes many references on the theory and application of SPSA. On this Web site, one can find many accounts of numerical studies that are consistent with the efficiency statement above. (Of course, given that the statement is based on asymptotic arguments and associated regularity conditions, one should not assume that the result always holds.) In addition, there are references describing many applications. These include queuing systems, pattern recognition, industrial quality improvement, aircraft design, simulation-based optimization, bioprocess control, neural network train- 
ing, chemical process control, fault detection, human-machine interaction, sensor placement and configuration, and vehicle traffic management.

We will not present the formal conditions for convergence and asymptotic normality of SPSA, as such conditions are available in many references (e.g., Dippon and Renz, 1997; Gerencsér, 1999; Spall, 1992, 2003, Chap. 7). These conditions are essentially identical to the standard conditions for convergence of SA algorithms, with the exception of the additional conditions on the user-generated perturbation vector $\Delta_{k}$.

The choice of the distribution for generating the $\Delta_{k}$ is important to the performance of the algorithm. The standard conditions for the elements $\Delta_{k i}$ of $\Delta_{k}$ are that the $\left\{\Delta_{k i}\right\}$ are independent for all $k, i$, identically distributed for all $i$ at each $k$, symmetrically distributed about zero and uniformly bounded in magnitude for all $k$. In addition, there is an important inverse moments condition:

$$
E\left(\left|\frac{1}{\Delta_{k i}}\right|^{2+2 \tau}\right) \leq C
$$

for some $\tau>0$ and $C>0$. The role of this condition is to control the variation of the elements of $\hat{\boldsymbol{g}}_{k}\left(\hat{\boldsymbol{\theta}}_{k}\right)$ (which have $\Delta_{k i}$ in the denominator). One simple and popular distribution that satisfies the inverse moments condition is the symmetric Bernoulli \pm 1 distribution. (In fact, as discussed in Spall, 2003, Sect. 7.7, this distribution can be shown to be optimal under general conditions when using asymptotic considerations.) Two common mean-zero distributions that do not satisfy the inverse moments condition are symmetric uniform and normal with mean zero. The failure of both of these distributions is a consequence of the amount of probability mass near zero. Exercise 7.3 in Spall (2003) illustrates the dramatic performance degradation that can occur through using distributions that violate the inverse moments condition.

As with any real-world implementation of stochastic optimization, there are important practical considerations when using SPSA. One is to attempt to define $\boldsymbol{\theta}$ so that the magnitudes of the $\boldsymbol{\theta}$ elements are similar to one another. This desire is apparent by noting that the magnitudes of all components in the perturbations $c_{k} \Delta_{k}$ are identical in the case where identical Bernoulli distributions are used. Although it is not always possible to choose the definition of the elements in $\boldsymbol{\theta}$, in most cases an analyst will have the flexibility to specify the units for $\boldsymbol{\theta}$ to ensure similar magnitudes. Another important consideration is the choice of the gains $a_{k}, c_{k}$. The principles described for FDSA above apply to SPSA as well. Section 7.5 of Spall (2003) provides additional practical guidance.

There have been a number of important extensions of the basic SPSA method represented by the combination of (6.4) and (6.6). Three such extensions are to the problem of global (versus local) optimization, to discrete (versus continuous) problems, and to include second-order-type information (Hessian matrix) with the aim of creating a stochastic analogue to the deterministic Newton-Raphson method. 
The use of SPSA for global minimization among multiple local minima is discussed in Maryak and Chin (2001). One of their approaches relies on injecting Monte Carlo noise in the right-hand side of the basic SPSA updating step in (6.4). This approach is a common way of converting SA algorithms to global optimizers through the additional "bounce" introduced into the algorithm (Yin, 1999). Maryak and Chin (2001) also show that basic SPSA without injected noise (i.e., (6.4) and (6.6) without modification) may, under certain conditions, be a global optimizer. Formal justification for this result follows because the random error in the SP gradient approximation acts in a way that is statistically equivalent to the injected noise mentioned above.

Discrete optimization problems (where $\boldsymbol{\theta}$ may take on discrete or combined discrete/continuous values) are discussed in Gerencsér et al. (1999). Discrete SPSA relies on a fixed-gain (constant $a_{k}$ and $c_{k}$ ) version of the standard SPSA method. The parameter estimates produced are constrained to lie on a discrete-valued grid. Although gradients do not exist in this setting, the approximation in (6.6) (appropriately modified) is still useful as an efficient measure of slope information.

Finally, using the simultaneous perturbation idea, it is possible to construct a simple method for estimating the Hessian (or Jacobian) matrix of $L$ while, concurrently, estimating the primary parameters of interest $(\theta)$. This adaptive SPSA (ASP) approach produces a stochastic analogue to the deterministic NewtonRaphson algorithm (e.g., Bazaraa et al., 1993, pp. 308-312), leading to a recursion that is optimal or near-optimal in its rate of convergence and asymptotic error. The approach applies in both the gradient-free setting emphasized in this section and in the root-finding/stochastic gradient-based (Robbins-Monro) setting reviewed in Spall (2003, Chaps. 4 and 5). Like the standard SPSA algorithm, the ASP algorithm requires only a small number of loss function (or gradient, if relevant) measurements per iteration - independent of the problem dimension - to adaptively estimate the Hessian and parameters of primary interest. Further information is available at Spall (2000) or Spall (2003, Sect. 7.8).

\subsection{Genetic Algorithms}

\subsubsection{Introduction}

Genetic algorithms (GAs) represent a popular approach to stochastic optimization, especially as relates to the global optimization problem of finding the best solution among multiple local mimima. (GAs may be used in general search problems that are not directly represented as stochastic optimization problems, but we focus here on their use in optimization.) GAs represent a special case of the more general class of evolutionary computation algorithms (which also includes methods such as evolutionary programming and evolution strategies). The GA applies when the elements of $\boldsymbol{\theta}$ are real-, discrete-, or complex-valued. As suggested by the name, the $\mathrm{GA}$ is based loosely on principles of natural evolution and survival of the fittest. 
In fact, in GA terminology, an equivalent maximization criterion, such as $-L(\boldsymbol{\theta})$ (or its analogue based on a bit-string form of $\boldsymbol{\theta}$ ), is often referred to as the fitness function to emphasize the evolutionary concept of the fittest of a species.

A fundamental difference between GAs and the random search and SA algorithms considered in Sects. 6.2 and 6.3 is that GAs work with a population of candidate solutions to the problem. The previous algorithms worked with one solution and moved toward the optimum by updating this one estimate. GAs simultaneously consider multiple candidate solutions to the problem of minimizing $L$ and iterate by moving this population of solutions toward a global optimum. The terms generation and iteration are used interchangeably to describe the process of transforming one population of solutions to another. Figure 6.3 illustrates the successful operations of a GA for a population of size 12 with problem dimension $p=2$. In this conceptual illustration, the population of solutions eventually come together at the global optimum.

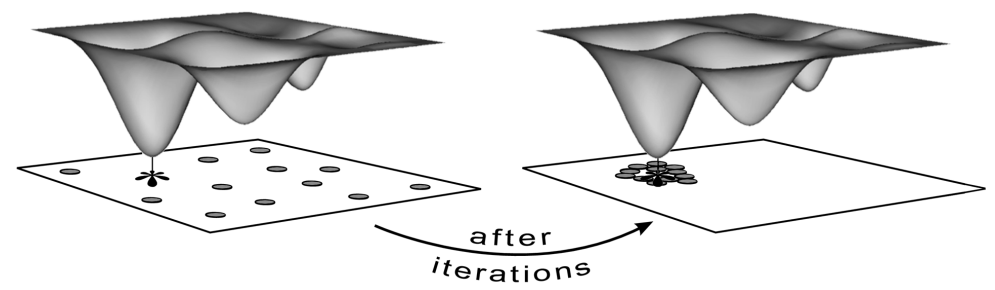

Figure 6.3. Minimization of multimodal loss function. Successful operations of a GA with a population of 12 candidate solutions clustering around the global minimum after some number of iterations (generations) (Reprinted from Introduction to Stochastic Search and Optimization with permission of John Wiley \& Sons, Inc.)

The use of a population versus a single solution affects in a basic way the range of practical problems that can be considered. In particular, GAs tend to be best suited to problems where the loss function evaluations are computer-based calculations such as complex function evaluations or simulations. This contrasts with the single-solution approaches discussed earlier, where the loss function evaluations may represent computer-based calculations or physical experiments. Population-based approaches are not generally feasible when working with realtime physical experiments. Implementing a GA with physical experiments requires that either there be multiple identical experimental setups (parallel processing) or that the single experimental apparatus be set to the same state prior to each population member's loss evaluation (serial processing). These situations do not occur often in practice.

Specific values of $\boldsymbol{\theta}$ in the population are referred to as chromosomes. The central idea in a GA is to move a set (population) of chromosomes from an initial collection of values to a point where the fitness function is optimized. We let $N$ denote the population size (number of chromosomes in the population). Most of the early work in the field came from those in the fields of computer science and artificial intelligence. More recently, interest has extended to essentially all 
branches of business, engineering, and science where search and optimization are of interest. The widespread interest in GAs appears to be due to the success in solving many difficult optimization problems. Unfortunately, to an extent greater than with other methods, some interest appears also to be due to a regrettable amount of "salesmanship" and exaggerated claims. (For example, in a recent software advertisement, the claim is made that the software "... uses GAs to solve any optimization problem." Such statements are provably false.) While GAs are important tools within stochastic optimization, there is no formal evidence of consistently superior performance - relative to other appropriate types of stochastic algorithms - in any broad, identifiable class of problems.

Let us now give a very brief historical account. The reader is directed to Goldberg (1989, Chap. 4), Mitchell (1996, Chap. 1), Michalewicz (1996, pp. 1-10), Fogel (2000, Chap. 3), and Spall (2003, Sect. 9.2) for more complete historical discussions. There had been some success in creating mathematical analogues of biological evolution for purposes of search and optimization since at least the 1950 s (e.g., Box, 1957). The cornerstones of modern evolutionary computation - evolution strategies, evolutionary programming, and GAs - were developed independently of each other in the $1960 \mathrm{~s}$ and $1970 \mathrm{~s}$. John Holland at the University of Michigan published the seminal monograph Adaptation in Natural and Artificial Systems (Holland, 1975). There was subsequently a sprinkle of publications, leading to the first full-fledged textbook Goldberg (1989). Activity in GAs grew rapidly beginning in the mid-1980 s, roughly coinciding with resurgent activity in other artificial intelligence-type areas such as neural networks and fuzzy logic. There are now many conferences and books in the area of evolutionary computation (especially GAs), together with countless other publications.

\subsubsection{Chromosome Coding and the Basic GA Operations}

This section summarizes some aspects of the encoding process for the population chromosomes and discusses the selection, elitism, crossover, and mutation operations. These operations are combined to produce the steps of the GA.

An essential aspect of GAs is the encoding of the $N$ values of $\boldsymbol{\theta}$ appearing in the population. This encoding is critical to the GA operations and the associated decoding to return to the natural problem space in $\boldsymbol{\theta}$. Standard binary $(0,1)$ bit strings have traditionally been the most common encoding method, but other methods include gray coding (which also uses $(0,1)$ strings, but differs in the way the bits are arranged) and basic computer-based floating-point representation of the real numbers in $\boldsymbol{\theta}$. This 10-character coding is often referred to as realnumber coding since it operates as if working with $\boldsymbol{\theta}$ directly. Based largely on successful numerical implementations, this natural representation of $\boldsymbol{\theta}$ has grown more popular over time. Details and further references on the above and other coding schemes are given in Michalewicz (1996, Chap. 5), Mitchell (1996, Sects. 5.2 and 5.3), Fogel (2000, Sects. 3.5 and 4.3), and Spall (2003, Sect. 9.3).

Let us now describe the basic operations mentioned above. For consistency with standard GA terminology, let us assume that $L(\boldsymbol{\theta})$ has been transformed 
to a fitness function with higher values being better. A common transformation is to simply set the fitness function to $-L(\boldsymbol{\theta})+C$, where $C \geq 0$ is a constant that ensures that the fitness function is nonnegative on $\Theta$ (nonnegativity is only required in some GA implementations). Hence, the operations below are described for a maximization problem. It is also assumed here that the fitness evaluations are noise-free. Unless otherwise noted, the operations below apply with any coding scheme for the chromosomes.

selection and elitism steps occur after evaluating the fitness function for the current population of chromosomes. A subset of chromosomes is selected to use as parents for the succeeding generation. This operation is where the survival of the fittest principle arises, as the parents are chosen according to their fitness value. While the aim is to emphasize the fitter chromosomes in the selection process, it is important that not too much priority is given to the chromosomes with the highest fitness values early in the optimization process. Too much emphasis of the fitter chromosomes may tend to reduce the diversity needed for an adequate search of the domain of interest, possibly causing premature convergence in a local optimum. Hence methods for selection allow with some nonzero probability the selection of chromosomes that are suboptimal.

Associated with the selection step is the optional "elitism" strategy, where the $N_{e}<N$ best chromosomes (as determined from their fitness evaluations) are placed directly into the next generation. This guarantees the preservation of the $N_{e}$ best chromosomes at each generation. Note that the elitist chromosomes in the original population are also eligible for selection and subsequent recombination.

As with the coding operation for $\boldsymbol{\theta}$, many schemes have been proposed for the selection process of choosing parents for subsequent recombination. One of the most popular methods is roulette wheel selection (also called fitness proportionate selection). In this selection method, the fitness functions must be nonnegative on $\Theta$. An individual's slice of a Monte Carlo-based roulette wheel is an area proportional to its fitness. The "wheel" is spun in a simulated fashion $N-N_{e}$ times and the parents are chosen based on where the pointer stops. Another popular approach is called tournament selection. In this method, chromosomes are compared in a "tournament," with the better chromosome being more likely to win. The tournament process is continued by sampling (with replacement) from the original population until a full complement of parents has been chosen. The most common tournament method is the binary approach, where one selects two pairs of chromosomes and chooses as the two parents the chromosome in each pair having the higher fitness value. Empirical evidence suggests that the tournament selection method often performs better than roulette selection. (Unlike tournament selection, roulette selection is very sensitive to the scaling of the fitness function.) Mitchell (1996, Sect. 5.4) provides a good survey of several other selection methods.

The crossover operation creates offspring of the pairs of parents from the selection step. A crossover probability $P_{c}$ is used to determine if the offspring will represent a blend of the chromosomes of the parents. If no crossover takes place, then the two offspring are clones of the two parents. If crossover does take 
place, then the two offspring are produced according to an interchange of parts of the chromosome structure of the two parents. Figure 6.4 illustrates this for the case of a ten-bit binary representation of the chromosomes. This example shows one-point crossover, where the bits appearing after one randomly chosen dividing (splice) point in the chromosome are interchanged. In general, one can have a number of splice points up to the number of bits in the chromosomes minus one, but one-point crossover appears to be the most commonly used.

Note that the crossover operator also applies directly with real-number coding since there is nothing directly connected to binary coding in crossover. All that is required are two lists of compatible symbols. For example, one-point crossover applied to the chromosomes ( $\theta$ values) $[6.7,-7.4,4.0,3.9 \mid 6.2,-1.5]$ and $[-3.8,5.3,9.2,-0.6 \mid 8.4,-5.1]$ yields the two children: $[6.7,-7.4,4.0,3.9,8.4,-5.1]$ and $[-3.8,5.3,9.2,-0.6,6.2,-1.5]$.

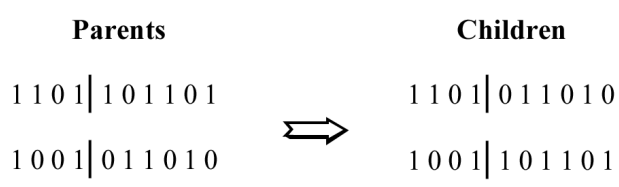

Figure 6.4. Example of crossover operator under binary coding with one splice point

The final operation we discuss is mutation. Because the initial population may not contain enough variability to find the solution via crossover operations alone, the GA also uses a mutation operator where the chromosomes are randomly changed. For the binary coding, the mutation is usually done on a bit-by-bit basis where a chosen bit is flipped from 0 to 1 , or vice versa. Mutation of a given bit occurs with small probability $P_{m}$. Real-number coding requires a different type of mutation operator. That is, with a $(0,1)$-based coding, an opposite is uniquely defined, but with a real number, there is no clearly defined opposite (e.g., it does not make sense to "flip" the 2.74 element). Probably the most common type of mutation operator is simply to add small independent normal (or other) random vectors to each of the chromosomes (the $\boldsymbol{\theta}$ values) in the population.

As discussed in Sect. 6.1.4, there is no easy way to know when a stochastic optimization algorithm has effectively converged to an optimum. this includes gas. The one obvious means of stopping a GA is to end the search when a budget of fitness (equivalently, loss) function evaluations has been spent. Alternatively, termination may be performed heuristically based on subjective and objective impressions about convergence. In the case where noise-free fitness measurements are available, criteria based on fitness evaluations may be most useful. for example, a fairly natural criterion suggested in Schwefel (1995, p. 145) is to stop when the maximum and minimum fitness values over the $N$ population values within a generation are sufficiently close to one another. however, this criterion provides no formal guarantee that the algorithm has found a global solution. 


\section{The Core Genetic Algorithm}

The steps of a basic form of the GA are given below. These steps are general enough to govern many (perhaps most) modern implementations of GAs, including those in modern commercial software. Of course, the performance of a GA typically depends greatly on the implementation details, just as with other stochastic optimization algorithms. Some of these practical implementation issues are taken up in the next section.

\section{Core GA Steps for Noise-Free Fitness Evaluations}

Step o (Initialization) Randomly generate an initial population of $N$ chromosomes and evaluate the fitness function (the conversion of $L(\boldsymbol{\theta})$ to a function to be maximized for the encoded version of $\boldsymbol{\theta}$ ) for each of the chromosomes.

Step 1 (Parent Selection) Set $N_{e}=0$ if elitism strategy is not used; $0<N_{e}<N$ otherwise. Select with replacement $N-N_{e}$ parents from the full population (including the $N_{e}$ elitist elements). The parents are selected according to their fitness, with those chromosomes having a higher fitness value being selected more often.

Step 2 (Crossover) For each pair of parents identified in Step 1, perform crossover on the parents at a randomly (perhaps uniformly) chosen splice point (or points if using multi-point crossover) with probability $P_{c}$. If no crossover takes place (probability $1-P_{c}$ ), then form two offspring that are exact copies (clones) of the two parents.

Step 3 (Replacement and Mutation) While retaining the $N_{e}$ best chromosomes from the previous generation, replace the remaining $N-N_{e}$ chromosomes with the current population of offspring from Step 2. For the bit-based implementations, mutate the individual bits with probability $P_{m}$; for real coded implementations, use an alternative form of "small" modification (in either case, one has the option of choosing whether to make the $N_{e}$ elitist chromosomes candidates for mutation).

Step 4 (Fitness and End Test) Compute the fitness values for the new population of $N$ chromosomes. Terminate the algorithm if the stopping criterion is met or if the budget of fitness function evaluations is exhausted; else return to Step 1.

\section{Some Implementation Aspects}

While the above steps provide the broad outline for many modern implementations of GAs, there are some important implementation aspects that must be decided before a practical implementation. This section outlines a few of those aspects. More detailed discussions are given in Mitchell (1996, Chap. 5), Michalewicz (1996, Chaps. 4-6), Fogel (2000, Chaps. 3 and 4), Goldberg (2002, Chap. 12), and other references mentioned below. A countless number of numerical studies have been reported in the literature; we do not add to that list here. 
As with other stochastic optimization methods, the choice of algorithm-specific coefficients has a significant impact on performance. With GAs, there is a relatively large number of user decisions required. The following must be set: the choice of chromosome encoding, the population size $(N)$, the probability distribution generating the initial population, the strategy for parent selection (roulette wheel or otherwise), the number of splice points in the crossover, the crossover probability $\left(P_{c}\right)$, the mutation probability $\left(P_{m}\right)$, the number of retained chromosomes in elitism $\left(N_{e}\right)$, and some termination criterion. Some typical values for these quantities are discussed, for example, in Mitchell (1996, pp. 175-177) and Spall (2003, Sect. 9.6).

Constraints on $L(\boldsymbol{\theta})$ (or the equivalent fitness function) and/or $\boldsymbol{\theta}$ are of major importance in practice. The bit-based implementation of GAs provide a natural way of implementing component-wise lower and upper bounds on the elements of $\boldsymbol{\theta}$ (i.e., a hypercube constraint). More general approaches to handling constraints are discussed in Michalewicz (1996, Chap. 7 and Sects. 4.5 and 15.3) and Michalewicz and Fogel (2000, Chap. 9).

Until now, it has been assumed that the fitness function is observed without noise. One of the two possible defining characteristics of stochastic optimization, however, is optimization with noise in the function measurements (Property I in Sect. 6.1.3). There appears to be relatively little formal analysis of GAs in the presence of noise, although the application and testing of GAs in such cases has been carried out since at least the mid-1970 s (e.g., De Jong, 1975, p. 203). A large number of numerical studies are in the literature (e.g., the references and studies in Spall, 2003, Sects. 9.6 and 9.7). As with other algorithms, there is a fundamental tradeoff of more accurate information for each function input (typically, via an averaging of the inputs) and fewer function inputs versus less accurate ("raw") information to the algorithm together with a greater number of inputs to the algorithm. There appears to be no rigorous comparison of GAs with other algorithms regarding relative robustness to noise. Regarding noise, Michalewicz and Fogel (2000, p. 325) state: "There really are no effective heuristics to guide the choices to be made that will work in general."

\subsubsection{Some Comments on the Theory for GAs}

One of the key innovations in Holland (1975) was the attempt to put GAs on a stronger theoretical footing than the previous ad hoc treatments. He did this by the introduction of schema theory. While many aspects and implications of schema theory have subsequently been challenged (Reeves and Rowe, 2003, Chap. 3; Spall, 2003, Sect. 10.3), some aspects remain viable. In particular, schema theory itself is generally correct (subject to a few modifications), although many of the assumed implications have not been correct. With the appropriate caveats and restrictions, schema theory provides some intuitive explanation for the good performance that is frequently observed with GAs.

More recently, Markov chains have been used to provide a formal structure for analyzing GAs. First, let us mention one negative result. Markov chains can be used 
to show that a canonical GA without elitism is (in general) provably nonconvergent (Rudolph, 1994). That is, with a GA that does not hold onto the best solution at each generation, there is the possibility (through crossover and mutation) that a chromosome corresponding to $\boldsymbol{\theta}^{*}$ will be lost. (Note that the GA without elitism corresponds to the form in Holland, 1975.)

On the other hand, conditions for the formal convergence of GAs to an optimal $\theta^{*}$ (or its coded equivalent) are presented in Vose (1999, Chaps. 13 and 14), Fogel (2000, Chap. 4), Reeves and Rowe (2003, Chap. 6), and Spall (2003, Sect. 10.5), among other references. Consider a binary bit-coded GA with a population size of $N$ and a string length of $B$ bits per chromosome. Then the total number of possible unique populations is:

$$
N_{P} \equiv\left(\begin{array}{c}
N+2^{B}-1 \\
N
\end{array}\right)=\frac{\left(N+2^{B}-1\right) !}{\left(2^{B}-1\right) ! N !}
$$

(Suzuki, 1995). It is possible to construct an $N_{P} \times N_{P}$ Markov transition matrix $\boldsymbol{P}$, where the $i$ th element is the probability of transitioning from the $i$ th population of $N$ chromosomes to the $j$ th population of the same size. These elements depend in a nontrivial way on $N$, the crossover rate, and the mutation rate; the number of elite chromosomes is assumed to be $N_{e}=1$ (Suzuki, 1995). Let $\boldsymbol{p}_{k}$ be an $N_{P} \times 1$ vector having $j$ th component $p_{k}(j)$ equal to the probability that the $k$ th generation will result in population $j, j=1,2, \ldots, N_{P}$. From basic Markov chain theory,

$$
\boldsymbol{p}_{k+1}^{T}=\boldsymbol{p}_{k}^{T} \boldsymbol{P}=\boldsymbol{p}_{0}^{T} \boldsymbol{P}^{k+1},
$$

where $\boldsymbol{p}_{0}$ is an initial probability distribution. If the chain is irreducible and ergodic (see, e.g., Spall, 2003, Appendix E), the limiting distribution of the GA (i.e., $\overline{\boldsymbol{p}}^{T}=$ $\left.\lim _{k \rightarrow \infty} \boldsymbol{p}_{k}^{T}=\lim _{k \rightarrow \infty} \boldsymbol{p}_{0}^{T} \boldsymbol{P}^{k}\right)$ exists and satisfies the stationarity equation $\overline{\boldsymbol{p}}^{T}=\overline{\boldsymbol{p}}^{T} \boldsymbol{P}$. (Recall from basic Markov chain theory that irreducibility indicates that any state may be reached from any other state after a finite number of steps.)

Suppose that $\boldsymbol{\theta}^{*}$ is unique (i.e., $\Theta^{*}$ is the singleton $\boldsymbol{\theta}^{*}$ ). Let $J \subseteq\left\{1,2, \ldots, N_{P}\right\}$ be the set of indices corresponding to the populations that contain at least one chromosome representing $\boldsymbol{\theta}^{*}$. So, for example, if $J=\left\{1,6, N_{P}-3\right\}$, then each of the three populations indexed by 1, 6 and $N_{P}-3$ contains at least one chromosome that, when decoded, is equal to $\boldsymbol{\theta}^{*}$. Under the above-mentioned assumptions of irreducibility and ergodicity, $\sum_{i \in J} \bar{p}_{i}=1$, where $\bar{p}_{i}$ is the ith element of $\overline{\boldsymbol{p}}$. Hence, a GA with $N_{e}=1$ and a transition matrix that is irreducible and ergodic converges in probability to $\boldsymbol{\theta}^{*}$.

To establish the fact of convergence alone, it may not be necessary to compute the $\boldsymbol{P}$ matrix. Rather, it suffices to know that the chain is irreducible and ergodic. (For example, Rudolph, 1997, p. 125, shows that the Markov chain approach yields convergence when $0<P_{m}<1$.) However, $\boldsymbol{P}$ must be explicitly computed to get the rate of convergence information that is available from $\boldsymbol{p}_{k}$. This is rarely possible in practice because the number of states in the Markov chain (and hence dimension of the Markov transition matrix) grows very rapidly with increases in the population size 
and/or the number of bits used in coding for the population elements. For example, in even a trivial problem of $N=B=6$, there are $\sim 10^{8}$ states and $\sim 10^{16}$ elements in the transition matrix; this problem is much smaller than any practical GA, which can easily have 50 to 100 population elements and 15 to 40 bits per population element (leading to well over $10^{100}$ states, with each element in the corresponding row and column in the transition matrix requiring significant computation).

\subsection{Concluding Remarks}

Stochastic optimization is a major branch of computational statistics. This paper has been a whirlwind tour through some important issues and methods in stochastic optimization. Stochastic optimization applies when there are noisy measurements of the criterion being optimized and/or there is an injected Monte Carlo randomness as part of the algorithm. Of necessity, we cover only a small fraction of available methods in this relatively brief review, although the methods described (random search, stochastic approximation, and genetic algorithms) are representative of a broad range of important and widely used algorithms. Further, the treatment here on the specific algorithms is relatively brief. In particular, the subjects covered in this paper of approximately 30 pages are treated in over 160 pages in Spall (2003, Chaps. 1-2, 6-7, and 9-10) and are given an even more detailed treatment in the many specialized books or other references.

There are many challenges to carrying out real-world optimization, including the presence of noise in the function evaluations, the difficulties in distinguishing a globally optimal solution from locally optimal solutions, the "curse of dimensionality," the difficulties associated with nontrivial constraints, and the lack of stationarity in the solution as a result of the conditions of the problem changing over time. Stochastic optimization methods are especially useful in treating some of these challenges. In particular, by definition, they are designed for noisy function evaluations. Further, when considering injected (Monte Carlo) randomness (property II in Sect. 6.1.3), certain stochastic optimization algorithms will (under conditions, of course) serve as global optimizers. That is, the injected randomness provides enough "bounce" to the algorithm to allow for escape from local minima en route to achieving a global minimum.

In summary, while classical deterministic optimization methods (linear and nonlinear programming) are effective for a range of problems, stochastic methods are able to handle many of the problems for which deterministic methods are inappropriate. It is hoped that this summary gives the reader a flavor of the issues, algorithms, and challenges in carrying out optimization in the face of stochastic effects.

Acknowledgements. I appreciate the helpful comments of Dr. Stacy Hill on a draft version of this paper. Funding was provided by the U. S. Navy (contract Nooo2498-D-8124) and the JHU/APL Independent Research and Development (IRAD) 
Program. Selected parts of this article have been reprinted, by permission, from J.C. Spall, Introduction to Stochastic Search and Optimization, (c)2003 by John Wiley and Sons, Inc.

\section{References}

Arsham, H. (1998), “Techniques for Monte Carlo Optimizing," Monte Carlo Methods and Applications, vol. 4, pp. 181-229.

Baba, N., Shoman, T., and Sawaragi, Y. (1977), "A Modified Convergence Theorem for a Random Optimization Method," Information Sciences, vol. 13, pp. 159-166.

Bazaraa, M.S., Sherali, H.D., and Shetty, C.M. (1993), Nonlinear Programming: Theory and Algorithms (2nd ed.), Wiley, New York.

Blum, J.R. (1954), "Multidimensional Stochastic Approximation Methods," Annals of Mathematical Statistics, vol. 25, pp. 737-744.

Box, G.E.P. (1957), "Evolutionary Operation: A Method for Increasing Industrial Productivity," Journal of the Royal Statistical Society, Ser. C., vol. 6, pp. 81-101.

De Jong, K.A. (1975), "An Analysis of the Behavior of a Class of Genetic Adaptive Systems," Ph.D. dissertation, University of Michigan, Ann Arbor, MI (University Microfilms no. 76-9381).

Dippon, J. and Renz, J. (1997), "Weighted Means in Stochastic Approximation of Minima," SIAM Journal of Control and Optimization, vol. 35, pp. 1811-1827.

Fabian, V. (1971), "Stochastic Approximation," in Optimizing Methods in Statistics (J.S. Rustigi, ed.), Academic Press, New York, pp. 439-470.

Fogel, D.B. (2000), Evolutionary Computation: Toward a New Philosophy of Machine Intelligence (2nd ed.), IEEE Press, Piscataway, NJ.

Fouskakis, D. and Draper, D. (2002), "Stochastic Optimization: A Review," International Statistical Review, vol. 70, pp. 315-349.

Fu, M.C. (2002), "Optimization for Simulation: Theory vs. Practice" (with discussion by S. Andradóttir, P. Glynn, and J.P. Kelly), INFORMS Journal on Computing, vol. 14, pp. 192-227.

Gentle, J.E. (2003), Random Number Generation and Monte Carlo Methods (2nd ed.), Springer-Verlag, New York.

Gerencsér, L. (1999), "Convergence Rate of Moments in Stochastic Approximation with Simultaneous Perturbation Gradient Approximation and Resetting," IEEE Transactions on Automatic Control, vol. 44, pp. 894-905.

Gerencsér, L., Hill, S.D., and Vágó, Z. (1999), "Fixed Gain SPSA for Discrete Optimization," in Proceedings of the IEEE Conference on Decision and Control, 7-10 December 1999, Phoenix, AZ, pp. 1791-1795.

Goldberg, D.E. (1989), Genetic Algorithms in Search, Optimization, and Machine Learning, Addison-Wesley, Reading, MA.

Gosavi, A. (2003), Simulation-Based Optimization: Parametric Optimization Techniques and Reinforcement Learning, Kluwer Academic, Boston.

Holland, J.H. (1975), Adaptation in Natural and Artificial Systems, University of Michigan Press, Ann Arbor, MI. 
Karnopp, D.C. (1963), "Random Search Techniques for Optimization Problems," Automatica, vol. 1, pp. 111-121.

Kiefer, J. and Wolfowitz, J. (1952), "Stochastic Estimation of a Regression Function," Annals of Mathematical Statistics, vol. 23, pp. 462-466.

Kolda, T.G., Lewis, R.M., and Torczon, V. (2003), "Optimization by Direct Search: New Perspectives on Some Classical and Modern Methods," SIAM Review, vol. 45, pp. 385-482.

Kushner, H.J. and Yin, G.G. (1997), Stochastic Approximation Algorithms and Applications, Springer-Verlag, New York.

Maryak, J.L. and Chin, D.C. (2001), "Global Random Optimization by Simultaneous Perturbation Stochastic Approximation," in Proceedings of the American Control Conference, 25-27 June 2001, Arlington, VA, pp. 756-762.

Matyas, J. (1965), "Random Optimization," Automation and Remote Control, vol. 26, pp. 244-251.

Michalewicz, Z. (1996), Genetic Algorithms + Data Structures = Evolution Programs (3rd ed.), Springer-Verlag, New York.

Michalewicz, Z. and Fogel, D.B. (2000), How to Solve It: Modern Heuristics, Springer-Verlag, New York.

Mitchell, M. (1996), An Introduction to Genetic Algorithms, MIT Press, Cambridge, MA.

Nelder, J.A. and Mead, R. (1965), "A Simplex Method for Function Minimization," The Computer Journal, vol. 7, pp. 308-313.

Pflug, G.Ch. (1996), Optimization of Stochastic Models: The Interface Between Simulation and Optimization, Kluwer Academic, Boston.

Reeves, C.R. and Rowe, J.E. (2003), Genetic Algorithms - Principles and Perspectives: A Guide to GA Theory, Kluwer Academic, Boston.

Robbins, H. and Monro, S. (1951), "A Stochastic Approximation Method," Annals of Mathematical Statistics, vol. 22, pp. 400-407.

Rudolph, G. (1994), "Convergence Analysis of Canonical Genetic Algorithms," IEEE Transactions on Neural Networks, vol. 5, pp. 96-101.

Rudolph, G. (1997), Convergence Properties of Evolutionary Algorithms, Verlag Kovac, Hamburg.

Ruppert, D. (1991), "Stochastic Approximation," in Handbook of Sequential Analysis (B.K. Ghosh and P.K. Sen, eds.), Marcel Dekker, New York, pp. 503-529.

Schwefel, H.-P. (1995), Evolution and Optimum Seeking, Wiley, New York.

Solis, F.J. and Wets, J.B. (1981), "Minimization by Random Search Techniques," Mathematics of Operations Research, vol. 6, pp. 19-30.

Spall, J.C. (1992), "Multivariate Stochastic Approximation Using a Simultaneous Perturbation Gradient Approximation," IEEE Transactions on Automatic Control, vol. 37, pp. 332-341.

Spall, J.C. (2000), "Adaptive Stochastic Approximation by the Simultaneous Perturbation Method,"IEEE Transactions on Automatic Control, vol. 45, pp. 18391853.

Spall, J.C. (2003), Introduction to Stochastic Search and Optimization: Estimation, Simulation, and Control, Wiley, Hoboken, NJ. 
Suzuki, J. (1995), "A Markov Chain Analysis on Simple Genetic Algorithms," IEEE Transactions on Systems, Man, and Cybernetics, vol. 25, pp. 655-659.

Wolpert, D.H. and Macready, W.G. (1997), "No Free Lunch Theorems for Optimization," IEEE Transactions on Evolutionary Computation, vol. 1, pp. 67-82.

Yin, G. (1999), "Rates of Convergence for a Class of Global Stochastic Optimization Algorithms," SIAM Journal on Optimization, vol. 10, pp. 99-120.

Zhigljavsky, A.A. (1991), Theory of Global Random Search, Kluwer Academic, Boston. 